ISSN 0974-3618 (Print) 0974-360X (Online) www.rjptonline.org

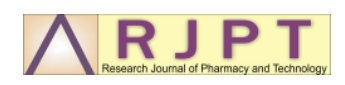

RESEARCH ARTICLE

\title{
Quantification Molecular Weight of Human Beta Defensin-2 and Human Beta Defensin-3 from Saliva of Caries patient
}

\author{
Retno Indrawati ${ }^{1 *}$, Indeswati Diyatri ${ }^{1}$, Fianza Rezkita $^{2}$, Rima Syafira Humaidy ${ }^{2}$ \\ ${ }^{1}$ Departement of Oral Biology, Faculty of Dental Medicine, Airlangga University, Surabaya - Indonesia. \\ ${ }^{2}$ Undergraduate Student, Faculty of Dental Medicine, Airlangga University, Surabaya - Indonesia. \\ *Corresponding Author E-mail: retnoindrawati@fkg.unair.ac.id
}

\begin{abstract}
:
Background: Dental caries is the most common multifactorial disease in the world. In saliva of caries patients, occur an increase in innate immunity, such as human beta defensin-2 (HBD-3) and human beta defensin-2 (HBD-3). Research on quantification the molecular weight of HBD-2 and HBD-3 in saliva of caries patients has not done yet. Objective: Determine the molecular weight from HBD-2 and HBD-3 from saliva of caries patients using the Western Blot method. Method: This study was approved by the Health Research Ethical Clearance Commission of Faculty of Dental Medicine Airlangga University No.156/HRECC.FODM/IV/2019. After every individual agreed, saliva sample from 16 children with caries and caries-free in the Dental Hospital Airlangga University was collected in June to July 2019. Male/female aged 9-12 years and DMF-t > 5, taken $5 \mathrm{ml}$ of saliva between 8-10 o'clock, passive-drool method without stimulation. Salivary samples were centrifuged for 20 minutes, $4^{\circ} \mathrm{C}$, with $10.000 \mathrm{rpm}$. Total protein was calculated using bicinchoninic acid (BCA) Assays before loading into Sodium Dodecyl Sulfate PolyAcrilamide Gel Electrophoresis (SDS-PAGE) to evaluate protein separation based on molecular weight. Furthermore, Western Blot is performed to ensure the protein that appears is HBD-2 and HBD-3 specific protein using primary and secondary antibodies. Results: THE molecular weight of HBD-2 and HBD-3 in the saliva of caries patients is $43 \mathrm{kDa}$, while the salivary free-caries band in the gel is unreadable, thus the molecular weight cannot be determined. Conclusion: The molecular weight of HBD-2 and HBD-3 in the saliva of caries patients calculated using the Western Blot method was $43 \mathrm{kDa}$.
\end{abstract}

KEYWORDS: Innate Immunity, Protein, Saliva, SDS-PAGE, Western Blot.

\section{INTRODUCTION:}

Dental caries is the most common multifactorial oral disease in the world and it increasing in the developing countries $^{1}$. Dental caries has three major factor which are bacteria, host, and diet related, but the cariogenic bacteria (S. mutans) has the major role to forms biofilm that could lead to dental caries and tooth $\operatorname{loss}^{2,3}$. Caries among children has negative effects such as disruption in their growth and development, emotional, and cognitive development ${ }^{4}$. Nowadays, researchers more focus on prevention (such as using oil pullling) ${ }^{5}$ and detection for dental caries instead of elimination with surgical approach $^{6}$.
There are various studies on to detect caries, one of which is using fluorescence, but it has disadvantages such as the ability to detect caries on the occlusal surface only ${ }^{7}$. The development of nanotechnology continues to increase with the times, the use of saliva as an early detection of caries began to be developed. Caries Saliva has function in caries protection by buffering the oral cavity, thus the atmosphere of the oral cavity is not too acidic and saliva also help in the remineralization of teeth $^{8}$. Anion in saliva has potentioal to be biomarker studies 9 . Saliva as diagnostic fluid able to detect various disease such as cancer (early diagnose), viruses, periodontitis, and hepatitis $\mathrm{C}^{10,11,12}$. The use of saliva as a diagnostic fluid of a disease has many advantages because it is considered non-invasive, easy, inexpensive, and safer. Saliva contains antimicrobial peptide that can be investigated namely human beta defensin (HBD) which plays a role in the salivary defense system. 
HBD is secreted due to the influence of proinflammatory mediators that appear as a form of resistance to pathogens in teeth with caries. There are several types of HBD from 1-6, but based on preliminary research that has been done before, HBD-2 and HBD-3 in the saliva of children who have caries is detected higher than HBD-1. Also, it has a broad spectrum of antibacterial activity on gram-negative and gram-positive. HBD-2 and HBD-3 has the ability to enhance the adaptive immune system in various ways, it can act as a chemoattractant for $\mathrm{B}$ cells, $\mathrm{T}$ cells, neutrophils, macrophages, and immature dendritic cells thus it implies that high levels of HBD-2 and HBD-3 in saliva have a role in the protection of mucous cavities mouth and teeth ${ }^{13}$. This indicates that caries will induce antimicrobial action, hence when caries occurs, HBD-3 levels will also be high in saliva.

The increase in HBD-2 and HBD-3 levels in saliva of caries patients is related to the molecular weight of HBD-3, thus this study aims to reveal the molecular weight of HBD-2 and HBD-3 derived from the saliva of caries patients using SDS-PAGE and Western Blot.

\section{MATERIAL AND METHODS: Sampling collection:}

Patients were instructed not to eat (only drink water) about 1 hour before collecting the saliva. Salivary withdrawal began with the patient gargled for about 0.5 minutes then let stand for 5 minutes. Collecting was done around 8 to 10 o'clock in the morning, adjusted to the circadian rhythm with passive drool without stimulation method (by bowing the head and saliva collected in the mouth, and let saliva to flow into a sterile centrifugation tube). Saliva was collected until 5 $\mathrm{ml}^{14,15}$.

Saliva samples that have been obtained are stored in an ice box after which the samples are moved to a refrigerator at $-30^{\circ} \mathrm{C}$ so that the samples last for a long period of time. Saliva samples that have been collected are immediately processed in the laboratory by being transferred to sterile tubes of $1 \mathrm{ml}$ size and then centrifuged for 20 minutes at $4^{\circ} \mathrm{C}$ for $10.000 \mathrm{rpm}$, then stored in a refrigerator with a temperature of $-70^{\circ} \mathrm{C}$ until further used for Western Blot ${ }^{16,17}$

\section{BCA Protein Assays:}

Procedure used is based on the Pierce TM BCA Protein Assays Kit protocol. The initial step is Reagent A $(10,000 \mu 1)$ and B $(200 \mu 1)$ mixed. Subsequently, the sample $(10 \mu \mathrm{l})$ was mixed with a mixture of reagents $\mathrm{A}$ and $\mathrm{B}(200 \mu \mathrm{l})$ and then incubated 30 minutes, $37^{\circ} \mathrm{C}$. After that it was put on a spectrophotometer with $562 \mathrm{~nm}$ for 10 minutes.

\section{SDS-PAGE:}

Based on the Bio-Rad Mini-PROTEAN ${ }^{\circledR}$ TGX ${ }^{\mathrm{TM}}$ protocol. Saliva samples $(15 \mu \mathrm{l})$ were mixed with SDS-

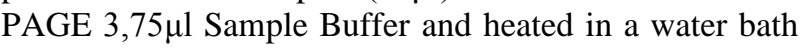
with $95^{\circ} \mathrm{C}$ for 5 minutes. An electroporator is prepared and samples were inserted into each well and a standard SeeBlue ${ }^{\circledR}$ Pre-stained protein marker with markers of 9$128 \mathrm{kDa}$ is inserted using a micropipette. Running SDSPAGE using 97 Volt and Ampere for 1 hour at 0.03 Ampere. Sample was staining with Coomosie Brilliant Blue and destaining with methanol for 24 hours. Samples washed with ddH20. Then, interpreted the gel.

\section{Western Blot:}

Based on the Bio-Rad Mini-PROTEAN® TGX TM protocol. The electrophoresis step is the same as SDSPAGE. Samples from caries and caries-free groups were put into electroporator with 114 Volt and 0.02 Ampere for 1 hour. The gel is transferred to the PVDF membrane (blotting process) with sandwich technique for 27 minutes with 0.1 Ampere and 4 Volts. Then, blocking process with skim milk $4^{\circ} \mathrm{C}$, overnight. Adding $100 \mu \mathrm{l}$ of primary antibody Beta Defensin 2 (2-RY-8): sc-134314 Santa Cruz Biotechnology then adding $5 \mu 1$ secondary antibody Anti-IgG HRP for HBD-2. Adding Beta defensin 103A (DEFB103A) (AA 6-22) antibody, Antibodies-online ${ }^{\circledR}$ (Primary antibody HBD-3) of 1: 100 $(50 \mu 1)$ then adding $5 \mu 1$ secondary antibody Anti-IgG HRP for HBD-3. Membrane in staining substrate ratio: ECL 1: 1 (each $3 \mathrm{ml}$ ). The membrane is interpreted and calculated using the molecular weight estimation protocol by Bio-Rad Laboratories.

\section{RESULTS:}

On examination determined BCA Protein Assay samples to be onloading to SDS-PAGE and Western blot was $15 \mu l$. The SDS-PAGE results (figure 1) show that in the gel all samples (16 samples) have bands which indicate that each sample has a protein with a molecular weight and readable. It can be seen from Figure 1 that several lines appear on the gel. Protein Marker uses standard SeeBlue ${ }^{\circledR}$ Pre-stained protein. From the results of this SDS-PAGE, we can proceed to the next stage which is Western Blot. 

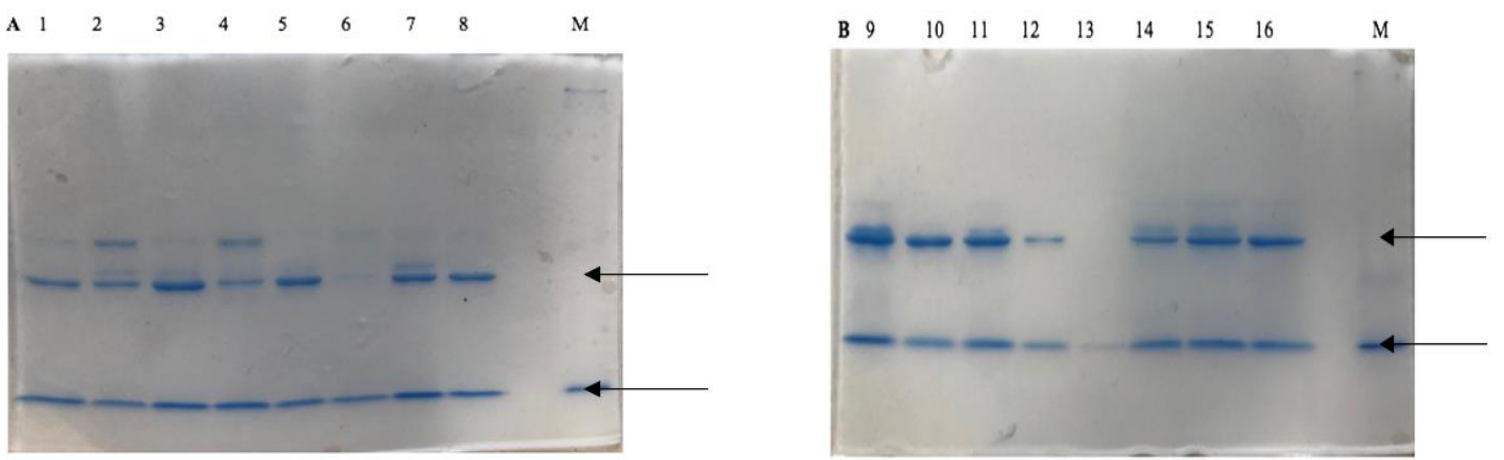

Figure 1. Samples SDS-PAGE. a) samples 1-8; b) samples 9-16. $M$ as marker.

HBD-2 and HBD-3 results in Western Blot method from caries and caries-free samples are exemplified from the highest DMF-t samples, as shown below (images from other samples we did not include). The first band is sample number 11 with DMF-t index value of 11 and the second band is sample number 7 with DMF-t index 12 (figure 2). Both, HBD-2 and HBD-3 bands are between numbers 38-49 $\mathrm{kDa}$, thus it can be read that HBD-2 and HBD-3 in the saliva of caries patients with DMF-t height has a molecular weight ranging from $38-49 \mathrm{kDa}$. In the control (sample free caries) no band appeared on the gel.

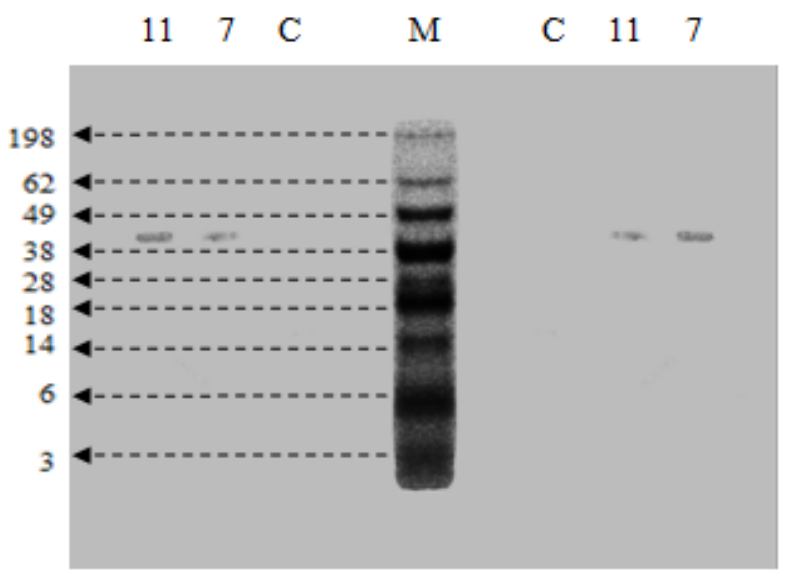

Figure 2. Samples Western Blot. Sample number 11 is saliva with DMF-t index value of 11 and the second band is sample number 7 with DMF-t index 12 and sample $C$ is sample with free caries. $M$ as Marker with number 198 kDa, 62 kDa, 49 kDa, 38 kDa, 28 kDa, 18 kDa, 14 kDa, 6 kDa, dan 3 kDa.

Furthermore, the calculation of molecular weight uses a linear equation by determining the retardation factor (Rf), which is the comparison of the migration distance of the protein with the distance of the migration of the dye in each marker band and then calculates the log molecular weight. Based on the results of Rf and log molecular weights are made into curves with linear equations. This linear equation is obtained: $y=-0,276 x+$ 2,049 , where $\mathrm{Y}$ is the logarithm of molecular weight and $\mathrm{X}$ is the value of Rf. In the HBD-2 and HBD-3 bands of salivary with caries is in the same line, the Rf found was
$1.5 \mathrm{~cm}$, hence if it was entered into the formula $\mathrm{y}=$ $0.276(1.5)+2.049$ then $y=1.635$. Next, to find the molecular weight is to calculate the antilog value of $y$. Based on calculations, the antilog of 1,635 is 43,1519077 or can be rounded to 43 . Thus, the molecular weight of HBD-2 and HBD-3 is 43. In the saliva of freecaries patients' band do not appear at all, hence molecular weight cannot be calculated.

\section{DISCUSSION:}

The sample used in this study was salivary caries patients with DMF-t above 5. Salivary samples were selected in children aged 9-12 because at that age children were susceptible to caries. Sampling is limited from 8 to 10 adjusting the circadian rhythm because at that hour the flow rate salivary is higher than at night ${ }^{18}$. The child is not sick or taking drugs to avoid biased results due to a suppressed immune condition.

In this study, BCA Assays is used to determine the total protein present in the sample in order to know the minimum amount of protein that can appear when smoothed in SDS-PAGE. SDS-PAGE was used to separate the molecular weight of saliva samples, but the band that appears is not specific. Then Western Blot method is used to ensure the band seen in the gel is a specific protein HBD-2 and HBD-3. The Western Blot method uses primary antibodies namely Beta Defensin 2 (2-RY-8): sc-134314 Santa Cruz Biotechnology for HBD-2 and Beta defensins 103A (DEFB103A) (AA 622) monoclonal antibodies for HBD-3 that aim to bind HBD-2 and HBD-3in the sample and use secondary antibodies namely Anti-IgG HRP as a significant enhancer of signal amplification, thus the color in the band more clearly ${ }^{19}$. In the Western Blot, this study uses monoclonal antibodies to produce specific protein binding.

In the Western Blot results of the two caries patients samples, a band appeared indicating that HBD-3 was expressed in the saliva of the caries patients. Whereas, in the free caries sample is no band appeared, this indicated 
that HBD-2 and HBD-3in the saliva of free-caries patients very small compared to HBD-2 and HBD-3in the saliva sample of caries patients. Under normal circumstances HBD-2 and HBD-3 remains secreted as a form of innate immunity that aims to maintain the integrity of the epithelium and fight commensal bacteria. HBD-2 and HBD-3has high cationic residue molecules in the form of lysine and arginine which are stabilized by a hydrogen bond, thus HBD-3 is able to create a stable dimer which is the reason for the antibacterial activity mechanism of HBD-2 and HBD- $3^{20}$. In caries condition, HBD-2 and HBD-3 acts as a communicator between innate immunity and adaptive immunity. The mechanism of HBD-2 and HBD-3 as a communicator is caries caused by $S$. Mutans that causing inflammation then the body secretes pro-inflammatory cytokines and dendritic cells which will induce naive $\mathrm{CD}^{+}{ }^{+}$will proliferate and differentiate into TH17 cells. Th17 works with the help of mediation from Interleukin-17 (Il-17) which is an important cytokine in connecting innate immunity (acute inflammation) reactions with Tcells-mediated adaptive immunity reactions hence the immune response changes to adaptive ${ }^{21}$.

Based on various literature, defensin molecular weight ranges from 4-6 kDa and for $\mathrm{HBD}-3$ it has a molecular weight of $5.1 \mathrm{kDa}^{17}$. However, in this study there was a significant increase in the molecular weight of HBD-3 in the range of $43 \mathrm{kDa}$. It is possible that peptide molecule binding to each other with sulfide bridge and interchain cysteine bond resulting in protein compaction. In addition, differences in molecular weight can occur due to chemical modification of proteins, especially glycosylation which can cause displacement band drastically ${ }^{22}$.

In this study, HBD-3 band in saliva of caries patients was more visible than HBD-3 in free-patients caries, thus HBD-3 in saliva could be used as a candidate for dental caries biomarkers.

\section{ACKNOWLEDGEMENT:}

The authors are grateful to the PDUPT funds from the Government of Indonesia

\section{CONFLICT OF INTEREST:}

The authors declare no conflict of interest.

\section{REFERENCES:}

1. Manupati Prasanth, Adarsh Krishna Capoor. Antimicrobial Effect of Mouthwashes: An In vitro study. Research J. Pharm. and Tech 6(6): June 2013; Page 662-66.

2. K. Vanmathi Selvi, S. Aruna, S. Rajeshkumar. Analysis of Bioactive metabolites from Azollapinnata against Dental caries. Research J. Pharm. and Tech. 2017; 10(6): 1891-1896.

3. Hae-Ryoung Park. Effect of Salivary Streptococci mutans and Lactobacilli levels after uptake of the Probiotic for Clinical Trial. Research J. Pharm. and Tech. 2017; 10(9): 2984-2988.
4. Suganya M, Yadavi Soondron, Mahadeva Rao US. Oral and Dietary Habits, and Immunological and Clinical Impacts on the Incidence of Dental Caries - A Review. Research J. Pharm. and Tech. 7(11): Nov. 2014 Page 1348-1353.

5. Swathi R., Geetha R.V. Oil Pulling and Oral Health: A Review. Research J. Pharm. and Tech. 6(12): Dec. 2013; Page 1400-1401.

6. B. John Rozar Raj, Pradeep. Remineralising Agents in Dentistry. Research J. Pharm. and Tech 2016; 9(10):1734-1736.

7. Mohanraj M, Prabhu V, Senthil R. Diagnostic methods for early detection of dental caries - A review. International Journal of Pedodontic Rehabilitation. 2016;1(1):29-36.

8. Yoshizawa J, Schafer C, Schafer J, Farrell J, Paster B, Wong D. Salivary Biomarkers: Toward Future Clinical and Diagnostic Utilities. Clinical Microbiology Reviews. 2013;26(4):781-791.

9. Jung-Eun Park, Jong-Hwa Jang. Development and Application of an Analytical Method for Salivary Inorganic Anion Quantification for the Diagnosis of Systemic and Oral Diseases. Research J. Pharm. and Tech 2018; 11(5):1820-1824.

10. Ruchika Agarwal, Lakshmi T.. Salivary Enzymes as Biomarkers for Periodontitis - An Update. Research J. Pharm. and Tech. 7(1): Jan. 2014; Page 98-100.

11. V. Padmaharish. Salivary Biomarkers of Oral Cancer - A Review. Research J. Pharm. and Tech. 2016; 9(7):1007-1012.

12. Aastha Sareen, Swastika Tandon, Aparna Ramachandran, Srimathi R. Saliva as A Diagnostic Tool for Detection of the Viruses: A Review. Research J. Pharm. and Tech 2018; 11(10): 4739-4743.

13. Roestamadji R, Wahjuningrum D, Luthfi M, Soesiawan A, Pradopo S. Increased Levels of Human B Defensin in Childs with Dental Caries. International Medical Device and Technology Conference 2017. 2017;:124-126.

14. Khurshid Z, Zohaib S, Najeeb S, Zafar M, Slowey P, Almas K. Human Saliva Collection Devices for Proteomics: An Update. International Journal of Molecular Sciences. 2016;17(6):846.

15. Navazesh M, Kumar S. Measuring salivary flow. The Journal of the American Dental Association. 2008;139:35S-40S.

16. Aro K, Wei F, Wong D, Tu M. Saliva Liquid Biopsy for Point-ofCare Applications. Frontiers in Public Health. 2017;5(5):77.

17. Ghosh S, Gerken T, Schneider K, Feng Z, McCormick T, Weinberg A. Quantification of Human -Defensin-2 and -3 in Body Fluids: Application for Studies of Innate Immunity. Clinical Chemistry. 2007;53(4):757-765.

18. Takasu N, Toichi M, Nakamura W. Importance of regular lifestyle with daytime bright light exposure on circadian rhythm sleepwake disorders in pervasive developmental disorders. Japanese Dental Science Review. 2011;47(2):141-149.

19. Bass J, Wilkinson D, Rankin D, Phillips B, Szewczyk N, Smith K et al. An overview of technical considerations for Western blotting applications to physiological research. Scandinavian Journal of Medicine and Science in Sports. 2016;27(1):4-25.

20. Pazgier M, Hoover D, Yang D, Lu W, Lubkowski J. Human $\beta$ defensins. Cellular and Molecular Life Sciences. 2006;63(11):1294-1313.

21. Abbas A, Lichtman A, Pillai S, Baker D, Baker A. Cellular and Molecular Immunology. 9th ed. Philadelphia: Elsevier; 2018.

22. Guan Y, Zhu Q, Huang D, Zhao S, Jan Lo L, Peng J. An equation to estimate the difference between theoretically predicted and SDS PAGE-displayed molecular weights for an acidic peptide. Scientific Reports. 2015;5(1). 